\title{
CÁLCULO DA CONSTANTE DE DUREZA EM SISTEMAS FERROMAGNÉTICOS
}

Ronaldo Mota

Departamento de Física. Centro de Ciēncias Naturais e Exatas. UFSM. Santa Maria, RS.

RESUMO

Um modelo de duas bandas para o magnetismo é usado para calcular a constante de dureza em sistemas ferromagnēticos. Uma ban da è estreita e degenerada, representando os elëtrons "quasi-locali zados". A segunda é larga contendo poucos elétrons itinerantes. A susceptibilidade dinâmica transversa é calculada na aproximação de fase randōnica (RPA). A partir dos pölos da susceptibilidade dois modos de ondas de spin são obtidos: um modo acústico e um modo ópti co. o valor da constante de dureza da onda de spin acústica é essen cial para satisfazer o critērio de estabilidade ferromagnētica em $T=0 \mathrm{~K}$. Mostra-se que levar em conta o aclopamento de intercâmbio inter-atōmico entre elētrons das bandas estreitas è necessário para que a constante de dureza assuma o valor experimental.

\section{SUMMARY}

MOTA, R. 1986. Stiffness constant in ferromagnetic systems. Ciência e Natura, $8: 7-13$.

A two-band model for the magnetism is used to calculate the stiffness constant in ferromagnetic systems. One band is narrow and degenerated, representing the "quasi-localized" electrons. The second one is a wide band containing very few itinerant electrons. The transverse dynamical susceptibility is calculated within the random-phase approximation (RPA). The value of the stiffness constant of the acoustical spin wave is essential to satisfy the criterion of ferromagnetic stability at $T=0 \mathrm{~K}$. From the poles of the suscep tibility two spin-wave branches are derived: one acoustic and one optic. It is shown that taking into account the interatomic exchange coupling between electrons of the narrow bands is necessary for the spin-wave stiffness constant to assume the experimental value.

I. INTRODUÇAOO

0 estado de menor energia de um sistema ferromagnético po de ser excitado de tal maneira que a densidade de spin eletrōnico local possa precessar em torno da direção de equilïbrio da magneti zação. Os modos normais dessas precessões são denominados ondas de spin.

Assim sendo, ondas de spin, tambēm chamadas magnons, são excitações elementares de metais ferromagnēticos que surgem além das 
excitações de partīculas simples jā tratadas por nōs anteriormente (Mota e Coutinho-Filho, 1). Tais excitações estão relacionadas aos pares ligados elétron-buraco e são caracterizados pela dispersão da da pela energia $w(\vec{q})$ como uma função de vetor de onda $\vec{q}$. Para pé quenos valores de $\vec{q}$, isto $\bar{e}$, magnons de grande comprimento de onda, a relaçäo de dispersäo para ferromagnetos è da forma

$$
w(\vec{q})=D q^{2}
$$

onde $D$ é a constante de dureza ("stiffness constant") da onda de spin.

Neste trabalho incluiremos na descrição do sistema o ter mo de interação de intercâmbio inter-atomico, de forma similar ao realizado por Yamada e Shimizu (2) no cālculo de ondas de spin para metais de transiçäo. O fato de termos optado pela inclusáo deste ter mo, e não de outro, estā associado à procura de uma solução estāvel para o estado fundamental ferromagnético do ferro em $T=0 \mathrm{~K}$. 0 ter mo de interaçäo Coulombiano inter-atómico tenderia a desfavorecer o ferromagnetismo, tornando o estado fundamental ferromagnético mais instāvel a inda.

Para tornar positivo o sinal da constante de dureza das ondas de spin, critērio de estabilidade para a soluçào ferromagné tica, existem dois mecanismos principais: a inclusão do termo de in tercâmbio inter-atômico e a consideração de hibridização com as ban das sp, como mostrado por Muniz (3). Optamos no presente trabalho pelo primeiro caminho, porèm, è provăvel que o segundo mecanismo tam bēm contribua no mesmo sentido no que diz respeito ao sinal de $D_{a c}$. Como ressaltado anteriormente, o problema do caminho escolnido é que o termo de interação de intercâmbio inter-atōmico è muito difī cil de ser calculado a priori, e portanto, o tratamento usual finda sendo quando possivel, o ajuste deste parâmetro afim de reproduzir o valor conhecido de $D_{a c}$.

\section{HAMILTONIANO MODELO}

o Hamiltoniano modelo pode ser escrito na forma

$H=\sum_{i j \sigma \alpha} T_{i j}^{\alpha} c_{i}^{\alpha} c_{j}^{\alpha}+1 / 2 \sum_{i \sigma \alpha} U_{\alpha} n_{i \alpha}{ }^{n_{i-\alpha}}-2 J_{I L} \sum_{i} \vec{S}_{i}^{I} \cdot \vec{S}_{i}^{L}-2 \sum_{i<j} \vec{S}_{i}^{L} \cdot \vec{S}_{j}^{L}$,

onde $c_{\mathbf{j} \sigma}^{\alpha}\left(c_{i \sigma}^{\alpha^{+}}\right)$é o operador aniquilação (criação) para um elétron de spin no sītio i e banda $\alpha, n_{i \sigma}^{\alpha}$ e $\vec{S}_{i}^{\alpha}$ são o número ocupação e ope radores de spin destes elētrons, respectivamente, Tij são a repre sentação de Wannier das bandas dos elētrons, $U_{\alpha}$ são os acoplamentos coulombianos intra-bandas, J IL è o acoplamento de intercāmbio entre elētrons itinerantes de uma banda lärga I (largura $\Delta$ ) e elētrons "quasi-localizados" de uma banda estreita degenerada L (largura $\ell$ ), e $J$ é o acopllamento de intercāmbio inter-atōmico entre elétrons da 
banda estreita.

A caracteristica mais importante de nosso modelo é que am bos os elétrons, itinerantes e quasi-localizados, são governados pe 10 mesmo nível de Fermi, uma característica a ser preservada em sis temas de elētrons ditinerantes.

Hamiltoniano similar a (1) foi utilizado por Mota e Couti nho-Filho (1) para descrever o modelo de duas bandas para o magne tismo do Fe e tendo sido obtido excelentes resultados em comparação com vārios dados experimentais, tanto para baixas como al tas tempe raturas.

Para o caso em que a largura da banda degenerada estreita se aproxima de zero, os elétrons quasi-localizados tornam-se estri tamente localizados e o spin $S=1$ localizado surge e o modelo tor na-se idêntico ao de Edwards (4).

III. ESPECTRO DE ONDAS DE SPIN

ondas de spin, entendidas como excitações coletivas do sistema, estäo associadas aos zeros do denominador da susceptibil $\underline{i}$ dade dinâmica transversa, a qual precisa portanto ser obtida in cialmente.

Para simplificar os cálculos assumiremos bandas do tipo parabōlicas e a aproximação de massa efetiva. Alëm disso, assumire mos estados de um elétron na banda $I$ e dois na banda degenerada $L$. $0 \mathrm{~s}$ valores de

$\left.\left\langle n^{\alpha}\right\rangle=1 / N \Sigma_{\sigma} n_{k \sigma}^{\alpha}\right\rangle$, i.e., $\left\langle n^{I}\right\rangle=0.5$ e $\left\langle n^{L}\right\rangle=2.0$,

são tomados de dados de aniquilaçäo de pósitron em Fe (Johnson, 5). 0 s remanescentes quatro elētrons pertencem às bandas $T_{2 g}$ e não são relevantes em nossa anālise. Da solução paramagnētica em $T=0$ en contramos a relação $\mathrm{m}_{\mathrm{I}}{ }^{*} \Delta=\mathrm{m}_{\mathrm{L}}^{*} \Delta=\left(9 q^{2} \sqrt{2}\right)^{2 / 3}(\mathrm{n} / \mathrm{a})^{2}$, onde a è a cons tante de rede do Fe bcc e a posição relativa das bandas è fixada. No estado ferromagnético em $T=0 \operatorname{temos} \sum_{\sigma} \sigma\left(\left\langle n_{\sigma}^{I}\right\rangle+\left\langle n_{\sigma}^{L}\right\rangle\right)=2.2$ e $\left\langle n_{\psi}^{L}\right\rangle=0$, resultando $\left\langle n_{\uparrow}^{L}\right\rangle=2.0,\left\langle n_{\uparrow}^{I}\right\rangle=0.35$ e $\left\langle n_{\downarrow}^{I}\right\rangle=0.15$.

os parâmetros sãe tais a colocar o nivel de Fermi acima da banda $L$ no estado ferromagnético $T=0$.

A susceptibilidade dinâmica do sistema pode então ser ex presso por

$x_{-+}(q, w)=\frac{\left[x_{-+}^{I}(\vec{q}, w)\right]^{-1}+\left[x_{-+}^{L}(\vec{q}, w)\right]^{-1}+2 J_{I L}}{\left[x_{-+}^{L}(\vec{q}, w)\right]^{-1}\left[x_{-+}^{I}(\vec{q}, w)\right]^{-1}-J_{I L}^{2}}$

onde

$\left[x_{-+}^{I}(\vec{q}, w)\right]^{-1}=\left[x_{-+}^{O I}(\vec{q}, w)\right]^{-1}-U_{I}$, 
$\left[x_{-+}^{L}(\vec{q}, w)\right]^{-1}=\left[x_{-+}^{O L}(\vec{q}, w)\right]^{-1}-\left[U_{L}+J(\vec{q})\right]$

e

$x_{-+}^{0 \alpha}(\vec{q}, w)=(1 / N) \sum_{\vec{k}}\left[\left(\left\langle n^{\alpha}>-n_{\vec{k}+q, \downarrow}^{\alpha}>\right) /\left(w+\varepsilon^{\alpha}-\varepsilon^{\alpha}-\Delta_{\alpha}\right)\right]\right.$,

com

$\Delta_{\alpha}=w_{0 \downarrow}^{\alpha}-w_{0 \uparrow}^{\alpha}$

sendo o "splitting" de intercâmbio entre as bandas.

Como mostrado por Herring (6), o zero no denominador da

eq (2) fornece o espectro de energias de excitações coletivas do sistema. Dessa maneira, os polos $x_{-+}(\vec{q}, w)$ geram dois modos de ondas de spin, resultando um modo acústico e um modo öptico. A dependēn cia em q das ondas de spin dos dois modos podem ser obtidas em or dem $q^{2}$.

A expressão resultante para o modo acūstico $\left(w_{a c}\right) \bar{e}$ simi lar à obtida por Yamada e Shimizu (2) utilizando o método de modos normais na aproximação RPA. E também concordante com a expressão ob tida por Adamowicz (7) para o caso em que a banda estreita é assum $\underline{i}$ da no limite a tômico $(l=0)$, representando os elétrons d estritamen te localizados.

$$
\begin{aligned}
& 0 \text { modo acústico resulta } \\
& w_{a c}=D_{a c} q^{2}
\end{aligned}
$$

onde $D_{a c}$, a constante de dureza das ondas de spin é expressa por

$$
\begin{aligned}
& D_{a c}=\frac{J_{0} a^{2} \Delta n_{L}^{2}}{n_{I}+\Delta n_{L}}+\frac{1}{3\left(\Delta n_{I}+\Delta n_{L}\right)} \Gamma_{\frac{1}{N}}^{\frac{1}{k}} \frac{n_{\vec{k} \uparrow}^{\frac{I}{k}}+n_{\vec{k} \downarrow}^{\frac{I}{k}}}{2} \nabla^{2} \varepsilon_{\vec{k}}^{I}+ \\
& -\frac{1}{N} \sum_{\vec{k}} \frac{n \frac{I}{k} \uparrow-n \frac{I}{\vec{k} \downarrow}}{\Delta_{I}}\left(\nabla \frac{\left.\varepsilon_{\vec{k}}^{I}\right)^{2}}{3\left(\Delta n_{I}+\Delta n_{L}\right)} \cdot\right. \\
& \cdot\left[\left(\frac{1}{N} \sum_{\vec{k}} \frac{n \frac{L}{k \uparrow}+n \frac{L}{k} \downarrow}{2} \nabla^{2} \varepsilon_{\vec{k}}^{L}-\frac{1}{N} \sum_{\vec{k}} \frac{n \frac{L}{k} \uparrow-n \frac{L}{k} \downarrow}{\Delta_{L}} \nabla \varepsilon_{\vec{k}}^{L}\right)^{2}\right]
\end{aligned}
$$

onde $J_{0} \bar{e}$ a constante de acoplamento entre vizinhos mais próximos e a $\bar{e}$ a constante de rede.

$$
\begin{aligned}
& 0 \text { modo öptico pode ser escrito na forma } \\
& w_{o p}=w_{o p}^{0}-D_{o p} q^{2} .
\end{aligned}
$$


Embora este modo ainda não tenha sido estudado em suficiente deta 1hes, existem evidēncias experimentais de que o mesmo existe e inte rage com o acustico para vetores de onda suficientemente grandes (Cooke et ali, 8).

IV. CÁlCULO dA CONSTANTE dE DUREzA

Utilizando a parametrização proposta neste trabalho, ob tém-se, a partir da eq. (4),

$$
\begin{aligned}
D_{a c} & =23.962 J_{0} 10^{-39}+\frac{1}{6.6}(0.009 \Delta+2.070 \ell+ \\
& \left.-\frac{10.950 \ell^{2}}{2.0 U_{L}+16 J_{0}+0.022 \Delta-0.02 U_{I}}\right) 10^{-39}
\end{aligned}
$$

sendo $D_{a c}$ expresso em $\mathrm{Jm}^{2}$ e $\ell, \Delta, U_{I}, U_{L}$ e $J_{0}$ em eV.

0 nosso interesse é encontrar um conjunto de parâmetros que satisfaça ao mesmo tempo o valor conhecido de $D_{\text {ac }}$ em $T=0$ para o ferro, $D_{a c}=0.5024 .10^{-39} \mathrm{Jm}^{2}$ (Strinafellow, 9), a partir da eq. (6) e outros resultados experimentais, tais como a temperatura cri tica de Curie $\left(T_{C}=1044 \mathrm{~K}\right)$ e o momento magnētico em $T=0 \mathrm{~K}\left(2.2 \mu_{B} /\right.$ à tomo).

A tabela I mostra o valor resultante de $D_{a c}$, em $T=0 K$, cal culado a partir da eq. (4), assumindo sem interação inter-atōmica. $\left(J_{0}=0\right)$, para cada conjunto de parāmetros satisfazendo os valores experimentais citados acima.

TABELA I - TABELA MOSTRANDO OS RESULTADOS NUMERICOS CBTIDOS PARA DaC EM $T=$ OK PARA CADA CONJUNTO DE PARAMMETROS CONCORDANTES COM OS RESULTADOS EXPERIMENTAIS. D ${ }_{a c}$ EM $\mathrm{J} \mathrm{m}^{2}$ E OS DEMAIS EM eV. $J_{0}=0$

\begin{tabular}{cccccc}
\hline$\ell$ & $\Delta$ & $U_{I}$ & $J_{I L}$ & $U_{L}$ & $D_{a c}\left(10^{-39}\right)$ \\
0.70 & 10.00 & 0.200 & 1.080 & 0.429 & -0.524 \\
1.00 & 10.00 & 0.200 & 1.080 & 0.626 & -0.803 \\
2.00 & 7.50 & 0.200 & 0.805 & 1.312 & -1.745 \\
\hline
\end{tabular}

Pode-se observar da Tab. I que para nenhum conjunto de pa râmetros abtēm-se valores positivos de $D_{a c}$, muito menos concordan tes com o valor experimental conhecido. O fato de termos encontrado valores negativos para $D_{a c}$ implica ser o estado fundamental ferro magnētico instāvel em $T=O K$ para quando não se toma em conta a in teração inter-atômica para o ferro.

Considerando o termo de acoplamento de intercâmbio interatōmico vārios conjuntos de parâmetros são obtidos, para os quais, 
sem este termo, não se obteve valores satisfatōrios para $D_{a c}$. A in trodução da interaçăo de intercâmbio inter-atōmico alimina a insta bilidade do estado fundamental ferromagnético em $T=O K$ para o nos so modelo. A Tab. I I mostra os resultados obtidos, a partir da eq. (4).

TABELA II - TABELA MOSTRANDO OS RESULTADOS NUMERICOS OBTIDOS PARA $D_{\text {aC }}$ EM $T=$ OK PARA CADA CONJUNTO DE PARÃMETROS CONCOR DANTES COM OS RESULTADOS EXPERIMENTAIS. DaC EM $\mathrm{Jm}^{2}$ e OS DEMAIS EM eV.

\begin{tabular}{ccccccc}
\hline \hline$\ell$ & $\Delta$ & $U_{\mathrm{I}}$ & $\mathrm{J}_{\mathrm{IL}}$ & $U_{\mathrm{L}}$ & $J_{0}$ & $\mathrm{D}_{\mathrm{ac}}\left(10^{-39}\right)$ \\
0.70 & 10.00 & 0.200 & 1.080 & 0.086 & 0.043 & 0.5024 \\
1.00 & 10.00 & 0.200 & 1.080 & 0.190 & 0.054 & 0.5024 \\
2.00 & 7.50 & 0.000 & 0.825 & 0.560 & 0.094 & 0.5024 \\
0.20 & 5.00 & 0.000 & 0.550 & 0.010 & 0.024 & 0.5024 \\
\hline
\end{tabular}

V. CONCLUSÖES

A contradição acerca da estabilidade do sistema em $T=0 \mathrm{~K}$ surge por estarmos trabalhando com dois critēerios diferentes. 0 pri meiro deles, muito usual, que nos gerou a temperatura crítica,oqual $\bar{e}$ essencialmente o critērio de Stoner modificado, e o segundo o cál culo da constante de dureza das ondas de spin, pelo qual o sistema ferromagnético somente seria estāvel para $D_{a c}>0$. Katsuki e Wohlfarth (9) trataram esta questão conferindo especial atenção à relação das propriedades magnēticas dos metais com os valores de Dac. Mostraram tambēm que è possivel que atravēs do critērio de Stoner o sistema apresentar estabilidade ferromagnētica, enquanto esse estado funda mental è realmente instāvel. Eles concluỉram ser o critērio da cons tante de dureza mais forte do que o de Stoner, e portanto a estabi lidade do sistema deve ser determinada pelo sinal de Dac, sendo es tāvel se $D_{a c}>0$ e instāvel se $D_{a c}<0$.

Outros trabalhos, como o de wakoh et al (10) aplicaram a teoria de Yamada e Shimizu (2), sem levar em conta o termo interatōmico, encontrando resultados negativos ou muito pequenos para $D_{a c}$. Outros trabalhos nesta 1 inha (Thompson e Myers, 11 ; Thompson e Mook, 12; George e Thompson, 13) tambëm não encontraram para o fer ro resultados satisfatōrios.

No presente trabalho, utilizando tēcnicas de funções de Green, as excitações de uma partīcula do sistema foram calculadas na aproximação Hartree-Fock e a susceptibilidade dinâmica transver sa do sistema foi calculada na aproximação RPA. 0 espectro de ondas de spin foi calculado obtendo-se dois modos em ordem $q^{2}$ para longo comprimento de onda, un modo acūstico e um modo óptico. 
Os cálculos da constante de dureza das ondas de spin fo ram realizados, utilizando o modelo proposto, a partir dos conjun tos de parāmetros concordantes com o valor do momento magnētico em $T=0 \mathrm{~K}$ e com o valor da temperatura critica.

Mostrou-se, por fim, que sem levarmos em conta os termos de interaçäo inter-atōmica nenhum conjunto de parâmetros razoāvel foi encontrado. A inclusão deste termo implicou em obtermos vārios conjuntos de parāmetros satisfatōrios, evidenciando portanto o pa pel fundamental desempenhado pela interaçäo inter-atômica para eli minar a suposta instabilidade do estado fundamental ferromagnético em $T=O K$ encontrada por alguns autores.

\section{AGRADECIMENTOS}

Este trabalho foi parcialmente financiado pelo Conselho Nacional de Desenvolvimento Cientîfico e Tecnolōgico (CNPq).

\section{BIBLIOGRAFIA CITADA}

1. MOTA, Ronaldo \& Coutinho-FILHO, M.D. Phys. Rev. B 33: 7724-7728. 1986; MOTA, Ronaldo \& COUTINHO-FILHO, M.D. Journal of Magnetism and Magnetic Materials 54: 987-988, 1986.

2. YAMADA, H. \& SHIMIZU, M. Journal of the Physical Society of Japan 22: $1404-1411,1967$.

3. MUNIZ, R.B. Tese de Doutorado, Departament of Mathematics, Imperial College of Science and Technology (näo publicada).

4. EDWARDS, D.M. 1.Phys. F12: 1789-1796, 1982.

5. JOHNSON: 0. Pnys, Stat. Sol.(b) 99: 745-756, 1980.

6. HERRING, C. Magnetism. New York, Academic Press, 1966. $470 \mathrm{p}$.

7. ADAMOWICZ, L. J. Phys. F. Metal Physics: 2401-2412, 1977.

8. COOK, J.F., LYNN, J.N., DAVIS, H.L. Phys Rev. B 21: 4118-4130, 1980.

9. KATSUKI, A. \& WOHLFARTH, E.P. ProC. ROy. SOC. 295: 182-193, 1966.

10. WAKOH, S., EDWARDS, D.M., WOHLFFRTH, B.P. Physique 32 C1: 1073$1084,1971$.

11. THOMPSON, E.D. \& IIYERS, J.J. Phys. Rev. 153:574-582, 1967.

12. THOMPSON, E.D. MOOK, H.A. J. Appl. Phys. 41: 1227-1236, 1970.

13. GEORGE, P.K. \& THOMPSON, E.D. Intern. J. Mag. 1: 35-43, 1970.

Recebido em outubro, 1986; aceito em outubro, 1986. 
